\section{Gastos governamentais do SUS com internações hospitalares por causas externas: análise no Estado de São Paulo, 2000}

\section{Current Direct Costs of Hospital Admissions Due to External Causes - Analysis in the State of São Paulo, 2000}

\author{
Maria Helena Prado de Mello Jorge \\ Departamento de Epidemiologia \\ Faculdade de Saúde Pública \\ Universidade de São Paulo \\ Av. Dr. Arnaldo, 715 \\ 01246-904, São Paulo - SP \\ mhpjorge@usp.br
}

\section{Maria Sumie Koizumi}

Escola de Enfermagem

Universidade de São Paulo

\section{Resumo}

Introdução: No Brasil, vem ocorrendo importante crescimento das causas externas, afetando a morbimortalidade da sua população. Essas causas impõem ônus econômicos e sociais elevados, incluindo gastos hospitalares. Objetivo: conhecer as internações por lesões e envenenamentos em hospitais próprios ou conveniados com o SUS no Estado de São Paulo, buscando determinar o custo dessas hospitalizações para o poder público, segundo algumas variáveis consideradas importantes. Material: internações hospitalares em hospitais próprios ou conveniados com o SUS, no ano 2000, obtidas no Banco de Dados do Datasus para o Brasil e o Estado de São Paulo. Resultados e conclusões: comparando causas naturais (excluídas gravidez, parto e puerpério) e causas externas, as últimas representaram menos de $10 \%$ do total de internações, tiveram permanência menor e gastaram cerca de $10 \%$ do valor total pago para todas as internações. Embora a permanência seja menor, os gastos do SUS medidos pelo custo-dia e gasto médio, foram superiores aos das internações por causas naturais. As causas mais freqüentes de internação foram quedas e acidentes de transporte e apresentaram custo-dia e gasto médio superior às médias obtidas. Segundo o desfecho, os que vieram a falecer durante a internação tiveram gastos triplicados se comparados aos que receberam alta hospitalar.

Palavras-chave: Custos de internação. Ferimentos e lesões. Epidemiologia. Morbidade. Mortalidade.

Conflito de interesses: $O$ Editor Científico da RBE, participa do grupo de coordenação do Projeto de Políticas Públicas financiado pela FAPESP, do qual resultou o presente artigo. Não participou do processo de julgamento que foi inteiramente conduzido, sob sigilo, por um dos Editores Adjuntos.

FAPESP - Programa de Pesquisas em Políticas Públicas - Processo 01/027680-6 
Introduction: Important growth in external causes has been occurring in Brazil and affects the morbimortality of its population. These causes impose heavy economic and social burdens, including hospital costs. Objective: to analyze the hospitalizations for injuries and poisonings in SUS or SUS-licensed hospitals in the state of São Paulo and to determine the cost of these hospitalizations to the government, taking some important variables into consideration. Method: hospitalizations in SUS hospitals or SUS-licensed hospitals in 2000: information obtained in the Datasus database for Brazil and the state of São Paulo. Results and conclusions: comparing natural causes - excluding pregnancy, delivery, and puerperium - and external causes, the latter make up around $10 \%$ of total hospitalizations, required shorter stay periods, and were responsible for around $10 \%$ of the total amount paid for all the hospitalizations. Although stay may be shorter, SUS expenditures measured by cost per day and average cost were higher than hospitalizations for natural causes. Most frequent causes of hospitalization were falls and transportation accidents; these had costper day and average cost higher than the averages obtained. With regard to discharge conditions, costs tripled for those who died during hospitalization, compared to those in condition to be discharged.

Key Words: Hospital costs. Injuries. Epidemiology. Morbidity. Mortality.

\section{Introdução}

O crescimento das causas externas como causa de morte na população brasileira ocorreu principalmente a partir da década de 80, quando estas passaram a ocupar a segunda posição dentre os óbitos por todas a causas. No Brasil, no ano 2000, ocorreram 118.367 mortes por essas causas, o que representou $12,5 \%$ do total de óbitos.

Mundialmente, as análises das causas externas vêm sendo feitas com freqüência utilizando dados de mortalidade, visto serem mais facilmente obtidos, e de forma sistematizada, além de apresentarem melhor qualidade da informação. Já a análise da morbidade por estas causas vem exigindo criações de grandes bancos de dados especificamente desenhados para essa finalidade.

O banco de dados do "Major Trauma Outcome Study-MTOS” ${ }^{1}$ dos Estados Unidos da América é reconhecido como o de maior impacto na análise da morbidade hospitalar por trauma. Os estudos dele decorrentes têm sido fundamentais para a avaliação da gravidade e das intervenções em trauma.

No Brasil, não há bancos de dados similares. Entretanto, os dados do Sistema de Internações Hospitalares do Sistema Único de Saúde (SIH/SUS) têm possibilitado importantes análises quanto à morbidade hospitalar. Cumpre enfatizar que, a partir de 1997, as internações relacionadas com lesões e envenenamentos (CID-10, cap. XIX) ${ }^{2}$ tiveram o acréscimo do tipo de causa externa como diagnóstico secundário* . Esta resolução foi de suma importância, particularmente por propiciar as bases para orientar as atividades de prevenção desses agravos.

Estudos anteriores utilizando os dados do SIH/SUS e enfocando o trauma craniano demonstraram que o perfil dos pacientes que são internados por essa causa é diferente daquele verificado na mortalidade por causas externas, e que a morbimortalidade precisa ser considerada nesses dois conjun-

\footnotetext{
* Portaria MS no 142 de 13 de novembro de 1997, dispões sobre o preenchimento da autorização de Internação Hospitalar AlH - em casos com quadro compatível com causas externas. Diário oficial da União, Brasília V. 135, n 222, pág. 26499, 17 de novembro de 1997, Secção I.
} 
tos, tanto na assistência em trauma como também nos programas que visam a sua prevenção $0^{3,4}$.

No geral, utilizando este banco de dados (SIH/SUS), tem sido verificado que nas internações hospitalares as quedas predominam, atingindo quase metade de todas as internações por causas externas. Os acidentes de transporte aparecem em segundo lugar com proporções próximas a $20 \%$, e as agressões atingem cerca de $6 \%$. Na mortalidade, o predomínio recai sobre os homicídios, seguidos pelos acidentes de transporte ${ }^{5,6}$.

O SIH/SUS foi concebido para operar o sistema de mapeamento de internação dos hospitais contratados e tem apresentado melhoras gradativas ao longo de sua existência.

Sua abrangência limita-se às internações no âmbito do SUS, excluindo, portanto, as que são custeadas diretamente ou cobertas por seguro-saúde. Segundo estimativas, esse sistema reúne informações sobre cerca de $70 \%$ das internações hospitalares do país ${ }^{7,8}$. Entretanto, apesar da limitação quantitativa e de haver ainda problemas quanto à qualidade de informações, alguns autores ${ }^{9}$ referem que as estatísticas hospitalares permitem um quadro quase completo da mortalidade mais grave da população, qual seja a que leva à hospitalização.

O documento básico do Sistema é a Autorização de Internação Hospitalar (AIH), que habilita a internação do paciente e gera valores para pagamento. AAIH é preenchida pelo estabelecimento hospitalar prestador do serviço e enviada ao gestor municipal/estadual para o processamento e demais cumprimentos de suas finalidades. Os dados disponíveis para análise são: dados cadastrais da unidade de atendimento; identificação do paciente; dados sobre o diagnóstico principal e secundário codificados pela Classificação Internacional de Doenças (CID-10); procedimentos realizados e valores pagos. Outra limitação é aquela relativa ao fato de que reinternações ou transferências do mesmo paciente para outros hospitais não são identificadas, o que pode resultar em contagens cumulativas. A despeito de mais este viés, entretanto, o estudo das internações, quanto às suas diferentes características, permite uma boa visão da morbidade da população.

A violência, entendida aqui como o conjunto de agravos externos, não naturais, impõe ônus humanos e econômicos a todos os países e custa, anualmente, bilhões de dólares em assistência à saúde, custos legais, absenteísmo e produtividade perdida. ${ }^{10}$

Refere Iunes ${ }^{11}$ que, apesar de os acidentes e a violência atingirem proporções quase epidêmicas no Brasil dos pontos de vista da mortalidade e da morbidade, são ainda incipientes os esforços para se tentar estimar o seu impacto econômico no país.

Do ponto de vista econômico, o custo produzido por qualquer problema de saúde pode ser classificado em duas grandes categorias: custos diretos e custos indiretos.

Os custos diretos dizem respeito aos custos médicos e não médicos "relacionados ao diagnóstico, tratamento, recuperação e reabilitação da doença; os médicos diretos são aqueles impostos por ações e prescrições médicas, enquanto que os não médicos seriam representados, por exemplo, pelos gastos com transporte de pessoas que acompanham o paciente ao médico."

Os custos indiretos referem-se à perda de produção e produtividade trazida pelo problema de saúde, como a perda de dias de trabalho e, por exemplo, a menor produtividade gerada por limitações físicas.

Pesquisa sobre custos, enfocando um tipo específico de causa externa, foi realizada pelo Instituto de Pesquisa Econômica Aplicada $^{12}$. Com metodologia especificamente desenhada para esta finalidade, os custos dos acidentes de trânsito nas aglomerações urbanas brasileiras foram analisados abrangendo custos diretos e indiretos.

Estudos sobre custos hospitalares por causas externas, de uma forma global e em âmbito nacional, são escassos na revisão bibliográfica dos últimos anos. Internações hospitalares analisando determinadas causas externas ou tipos de trauma e seus respectivos custos é que são mais freqüentemente encontrados. E esses estudos enfocam alguns aspectos específicos dos custos, relacionando-os com violências, em 
senso estrito, acidentes de transporte (acidentes de moto), quedas, trauma craniano, trauma craniano por arma de fogo ${ }^{13-19}$. Retratam, portanto, análises fragmentadas por recortes específicos da amostra selecionada ou dos tipos de causa externa ou trauma que determinaram as internações.

Embora se saiba da dificuldade de mensuração dos custos de qualquer doença, principalmente porque esses dados não estão disponíveis, uma primeira estimativa poderia ser feita, medindo os gastos hospitalares, a partir dos dados do SIH/SUS, com base nas AIH (Automatização de Interação Hospitalar), conforme sugerido e já testado por alguns autores ${ }^{11,20,21}$.

Este trabalho objetiva, pois, conhecer os gastos governamentais com as internações decorrentes de lesões e envenenamentos pagas pelo Sistema Único de Saúde - SUS no Estado de São Paulo, a partir do SIH/ SUS, ano 2000.

Mesmo conhecendo os problemas e as limitações dos dados e, conseqüentemente, do estudo, eles "permitem indicar o sentido e a dimensão geral de algumas das categorias do custo econômico dos traumatismos" ${ }^{11}$.

Os achados, no trabalho, poderão subsidiar processos de planejamento, gestão e avaliação de políticas de assistência médicohospitalar no âmbito do SUS e contribuir para programas de prevenção das causas externas, entre nós.

\section{Objetivos}

Conhecer as internações por lesões e envenenamentos em hospitais próprios ou conveniados com o SUS no Estado de São Paulo, buscando determinar os gastos governamentais (SUS) dessas hospitalizações, segundo algumas variáveis consideradas importantes.

\section{Metodologia}

\section{Material}

Internações hospitalares em hospitais próprios ou conveniados com o SUS, no ano
2.000, obtidas no Banco de Dados do Datasus $^{22}$ para o Brasil e o Estado de São Paulo, que se constitui, portanto na fonte dos dados básicos das tabelas e da figura.

\section{Etapas do trabalho}

- Seleção de internações: do total de internações foram selecionadas as referentes a pacientes com AIH tipo I, visando não incluir as internações de pacientes de longa permanência.

- Exclusão das internações de mulheres por doenças ligadas à gravidez, ao parto e ao puerpério (Capitulo XV da CID-10). Conforme recomendação de alguns autores ${ }^{10}$, para a análise das internações é necessário excluir o grupo de causas ligadas ao ciclo gravídico-puerperal em razão de que:

$>$ o parto não pode ser considerado doença;

$>$ se incluído, provocaria, no mínimo, um desbalanço quanto a sexo e idade nas análises epidemiológicas.

- Agrupamento das internações por lesões e envenenamentos. Embora regras internacionais ${ }^{2}$ determinem que as internações por essas causas devam estar codificadas no capítulo XIX da CID-10 (lesões e envenenamentos e algumas outras conseqüências de causas externas) algumas vezes a codificação é feita utilizando-se o Capítulo XX (Causas Externas de morbidade e de mortalidade), razão pela qual esses valores devem ser somados, perfazendo, portanto, "internações por causas externas".

- Variáveis estudadas:

$>$ Número de internações por causas naturais (Capítulos I a XIV e XVI a XVIII da CID-10) e por causas externas segundo tipo e natureza da lesão (respectivamente Capítulos XIX eXX da CID-10)

$>$ Tempo médio de permanência

$>$ Gastos (analisando, gasto médio por paciente e custo dia)

$>$ Tipo de saída (alta ou óbito) 


\section{Resultados e discussão}

As etapas utilizadas para a construção do Banco de Dados permitiram algumas considerações e conclusões importantes. A Figura 1, construída para retratar a situação das internações no Brasil e no Estado de São Paulo, mostram essa situação.

É importante salientar que, das internações por causas externas, em número não desprezível de casos (quase 10\% segundo dados brasileiros), as AIH estão codificadas em desconformidade com as regras internacionais. Na Figura 1 pode ser verificado que 629.829 pacientes internados por lesões tiveram seu diagnóstico principal codificado no capítulo XIX da CID-10; e em 64.132, nesse campo, estava codificado o tipo de causa externa que levou à lesão. Esta constatação é importante, na medida em que, se esses casos não forem considerados, estar-se-á perdendo parcela significativa de internações decorrentes de lesões.

Feita essa correção, verifica-se que, no Brasil, do total de 9.023.370 internações rea- lizadas no ano 2000 o percentual de pacientes hospitalizados por traumatismos ou envenenamentos representou, para o SUS, 7,7\% das internações por todas as causas, com exclusão dos partos: foram 693.961 hospitalizações por causas externas. No Estado de São Paulo, o total de internações por essas mesmas causas correspondeu a 9,8\% (168.364 casos), num total de 1.724 .047 internações por todas as causas.

O tempo de permanência no hospital pode ser contado em dias de internação e pela permanência média, ou tempo médio de permanência, esta representada por:

$\mathrm{TMP}=\frac{\text { total de dias de permanência }}{\text { total de pacientes internados }}$

O total de dias de permanência de pacientes SUS internados por causas naturais e por causas externas, foi no Brasil, respectivamente, 51.302 .118 e 3.453.629, e em São Paulo, respectivamente, 10.515.997 e 787.326.

Pelo exame dos seus dados fica evidente que, tanto no Brasil quanto em São Paulo, os pacientes hospitalizados por lesões decor-

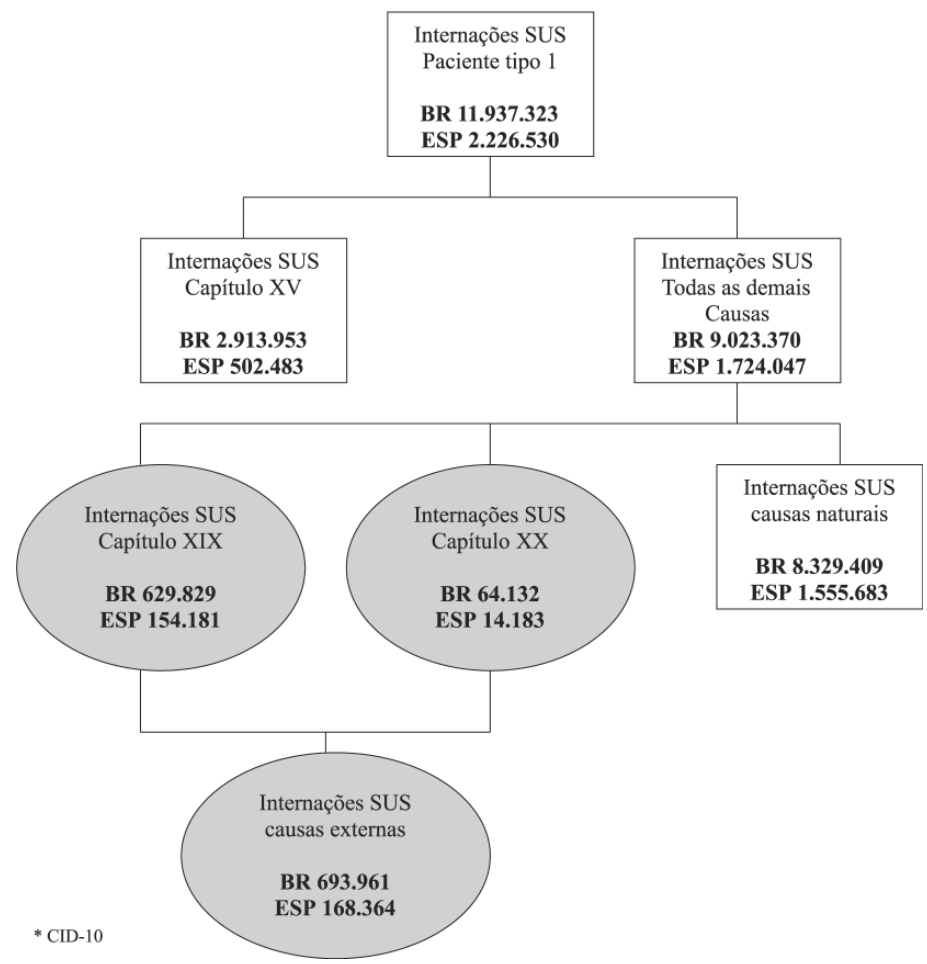

Figura 1 - Internações SUS segundo tipo de causa*, Brasil e Estado de São Paulo, 2000. Figure 1 - SUS hospital admissions according to cause*, Brazil and State of São Paulo, 2000. 
rentes de causas externas consomem entre 6 e $7 \%$ dos dias de permanência na instituição, em relação à permanência por todas as causas.

Medida segundo o tempo médio de permanência, a importância dos traumatismos e envenenamentos mostra-se menos importante, visto que os pacientes internados por causas naturais ficam, em média, 6,16 dias e 6,76 dias, respectivamente no Brasil e no Estado de São Paulo, e os pacientes internados por causas externas ficam em média 4,98 dias (dados para o Brasil) e 4,68 dias (dados para o Estado de São Paulo). (Tabela 1).

Os gastos do SUS com as internações hospitalares foram analisados segundo o gasto médio por paciente (GM) e o custodia (C-D), que corresponde na realidade ao valor pago pelo SUS por dia de internação, calculados por meio de:

$$
\begin{gathered}
\mathrm{GM}=\frac{\begin{array}{c}
\text { valor das despesas com internações } \\
\text { com causas externas }
\end{array}}{\begin{array}{c}
\text { número total de internações } \\
\text { por causas externas }
\end{array}} \\
\mathrm{C}-\mathrm{D}=\frac{\text { valor das despesas }}{\text { com causas externas }} \\
\begin{array}{c}
\text { número total de dias de permanência } \\
\text { por causas externas }
\end{array}
\end{gathered}
$$

Verifica-se que as internações por lesões (traumatismos/envenenamentos) têm gasto médio mais elevado para o seu tratamento do que aquelas em que o diagnóstico de internação tenha sido uma causa natural. Esse sobre-valor foi igual a $19,1 \%$ no Brasil e $4,4 \%$ no Estado de São Paulo.

Levando em conta que as internações por lesões têm menor tempo de permanência nos hospitais e custam mais caro ao SUS, a diferença provavelmente deve ser devida a procedimentos mais onerosos, cirurgias e/ ou diagnóstico por imagem. Justifica-se a análise deste assunto, visando a possibilidade de, contribuindo para a sua prevenção, se poder diminuir a demanda aos nossos hospitais.

Com relação ao custo-dia (em reais), é possível concluir, também, que este é mais elevado para as causas externas do que para as causas naturais (cerca de 50\%) (Tabela 1), conforme, aliás, é referido por Iunes, ao trabalhar com dados de $1997^{11}$.

Por se considerar importante, a esta altura, conhecer as causas mais freqüentes de internação, o tempo de internação e desfecho (alta ou óbito), e sua relação com gastos, apresenta-se a análise a seguir.

Alguns resultados merecem ser destacados. Em primeiro lugar, confirma-se que os

Tabela 1 - Internações SUS, tempo médio de permanência (em dias), gasto médio e custo-dia (em reais) segundo tipo de causa, Brasil e Estado de São Paulo, 2000.

Table 1 - SUS hospital admissions, average stay (in days), average expenditures and cost per day (in reais) according to cause, Brazil and State of São Paulo, 2000.

\begin{tabular}{lcc}
\hline & Brasil & Estado de São Paulo \\
\hline Internações SUS & & \\
Causas Naturais & $8.329 .409(92,3 \%)$ & $1.555 .683(90,2 \%)$ \\
Causas Externas & $693.961(7,7 \%)$ & $168.364(9,8 \%)$ \\
Total & $9.023 .370(100,0 \%)$ & $1.724 .047(100,0 \%)$ \\
\hline Tempo médio de permanência (em dias) & & \\
$\quad$ Causas Naturais & 6,16 & 6,76 \\
$\quad$ Causas Externas & 4,98 & 4,68 \\
\hline Gasto médio (em Reais) & & \\
$\quad$ Causas Naturais & 422,89 & 538,71 \\
$\quad$ Causas Externas & 503,70 & 562,24 \\
\hline Custo dia (em Reais) & & 79,69 \\
$\quad$ Causas Naturais & 68,66 & 120,23 \\
$\quad$ Causas Externas & 101,23 & \\
\hline
\end{tabular}


tipos de causas externas são distintos para a mortalidade e a morbidade hospitalar. $\mathrm{Na}$ mortalidade, conforme já referido, há predomínio dos homicídios, enquanto na morbidade hospitalar o destaque recai nas quedas, apontando dados importantes a serem assinalados nos programas de prevenção $0^{3,4,5,6}$.

Por outro lado, analisando-se essas internações pelo tipo de saída hospitalar, verifica-se que o grande predomínio das quedas $(50,0 \%)$, seguido dos acidentes de transporte (17,3\%), permanece somente naqueles que recebem alta. Nos que faleceram durante a internação, essa ordem encontrase mais atenuada nas quedas $(34,1 \%)$ eé proporcionalmente maior nos acidentes de transporte (26,1\%), indicando diferentes gravidades do trauma. Em relação às agressões, embora o percentual de internações seja menor $(5,7 \%)$, a freqüência dos que falecem é proporcionalmente maior, sinalizando também maior gravidade das lesões. Esse fato, aliás, já era esperado, na medida em que, em se tratando de lesão intencional provocada por terceira pessoa, o ânimo de matar deve evidenciar-se em lesão de maior gravidade. Com relação à mortalidade hospitalar segundo o tipo de causa externa (Tabela 2), pode-se verificar que a maior taxa é representada pelas tentativas de homicídio $(6,8 \%)$ e pelos acidentes com armas de fogo $(6,4 \%)$. As tentativas de suicídio foram responsáveis por uma mortalidade hospitalar de $4,9 \%$ e estão também entre os valores mais elevados da taxa.

A taxa de mortalidade hospitalar por lesões causadas por acidentes de transporte aparece em quarto $\left(4^{\circ}\right)$ lugar, com percentual de $4,7 \%$, seguida por queimaduras $(4,0 \%)$. Embora representando as quedas, como era esperado, o tipo mais freqüente de internação, a TMH, foi uma das mais baixas deste estudo $(2,1 \%)$.

Considerando que cerca de metade das internações foi devida a quedas, era de se esperar que os dias de internação de maior freqüência recaíssem também neste tipo de causa externa. De fato, as quedas totalizaram 352.297 dias, correspondendo a 43,64\% do

Tabela 2 - Internações SUS por causas externas ( $N^{\circ}$ e \%) e tempo médio de permanência - TMP - (em dias) e taxa de mortalidade hospitalar e de causa externa, ESP, 2000.

Table 2 - SUS hospital admissions due to external causes (N and \%) and average stay - TMP - (in days), and hospital and external cause mortality rates, State of São Paulo, 2000.

\begin{tabular}{lccccccc}
\hline Tipo & \multicolumn{2}{c}{ Alta } & \multicolumn{2}{c}{ Óbito } & \multicolumn{2}{c}{ Total } \\
& Internação & TMP & Internação & TMP & Internação & TMP & TMH \\
\hline Quedas & $84.155(50,5)$ & 4,03 & $1.829(34,1)$ & 7,39 & $85.984(50,0)$ & 4,1 & 2,1 \\
Acidentes de transporte & $28.406(17,0)$ & 5,76 & $1.397(26,1)$ & 5,13 & $29.803(17,3)$ & 5,7 & 4,7 \\
Envenenamento & $2.254(1,4)$ & 2,73 & $45(0,8)$ & 5,31 & $2.299(1,3)$ & 2,8 & 2,0 \\
Queimaduras & $2.775(1,7)$ & 7,38 & $117(2,2)$ & 12,51 & $2.892(1,7)$ & 7,6 & 4,0 \\
Penetração de & & & & & & & \\
Corpo estranho & $218(0,1)$ & 2,06 & $1(0,0)$ & 18,00 & $219(0,1)$ & 2,1 & 0,5 \\
Acidente por & & & & & & & \\
Arma de fogo & $3.438(2,1)$ & 5,25 & $237(4,4)$ & 2,81 & $3.675(2,1)$ & 5,1 & 6,4 \\
Demais acidentes & $20.816(12,5)$ & 4,78 & $501(9,3)$ & 7,22 & $21.317(12,4)$ & 4,8 & 2,4 \\
Suicídios e tentativas & $2.731(1,6)$ & 4,26 & $141(2,6)$ & 4,98 & $2.872(1,7)$ & 4,3 & 4,9 \\
Homicídios e tentativas & $9.060(5,4)$ & 5,51 & $666(12,4)$ & 3,79 & $9.726(5,7)$ & 5,4 & 6,8 \\
Ignorado & $6.599(4,0)$ & 4,43 & $168(3,1)$ & 6,61 & $6.767(3,9)$ & 4,5 & 2,5 \\
Seqüelas & $2.365(1,4)$ & 7,05 & $50(0,9)$ & 7,46 & $2.415(1,4)$ & 7,1 & 2,1 \\
Fatores suplementares & $3.811(2,3)$ & 5,20 & $208(3,9)$ & 7,44 & $4.019(2,3)$ & 5,3 & 5,2 \\
\hline Total & $166.628(100,0)$ & 4,65 & $5.360(100,0)$ & 6,15 & $171.988(100,0)$ & 4,7 & 3,1 \\
\hline
\end{tabular}

Nota: Os números entre parênteses referem-se às porcentagens.

Note: Numbers between parenthesis refer to percentage. 
total global de dias de permanência hospitalar por causas externas. Os acidentes de transporte, por sua vez, somaram 170.842 $(21,16 \%)$ dias de internação. Estas proporções ficaram mantidas tanto nos que receberam alta como nos que foram a óbito.

Conforme está demonstrado na Tabela 2, o tempo médio de permanência foi de 4,7 dias, similar aos que receberam alta. Nos que faleceram, o tempo médio foi mais elevado (6,2 dias).

Considerando os tipos de causas externas, algumas diferenças puderam ser detectadas. A menor média foi obtida em penetração de corpo estranho (2,1 dias) e a maior em seqüelas ( 7,1 dias).

Os tipos de saídas hospitalares também apresentaram algumas diferenças quando analisadas segundo os tipos de causas externas. Embora em geral o tempo médio tenha sido menor naquelas que obtiveram alta, alguns tipos de causas externas merecem ser destacados e comentados.

As internações por quedas apresentaram tempo médio de permanência abaixo da média (4,1 dias), mas, se considerado o tipo de saída, foi bastante alto naqueles que faleceram (7,4 dias). Na mesma lógica, os acidentes de transporte apresentaram média superior ao global (5,7 dias) e similaridade tanto nos que tiveram alta (5,8 dias) como naqueles que foram a óbito (5,1 dias).

Comportamento inverso foi constatado nos acidentes por arma de fogo e nos homicídios e tentativas de homicídio. Nestes, o tempo médio de permanência hospitalar dos pacientes que vieram a falecer foi menor, indicando a possibilidade de maior gravidade das lesões. É preciso lembrar ainda que a maioria das mortes por homicídio ocorre no próprio local do evento e a vítima sequer chega a receber atendimento hospitalar.

Distribuídos segundo os tipos de causas externas e a natureza das lesões, foram calculados os gastos médios e o custo-dia destas internações, tendo como base os valores pagos pelo SUS.

Considerado sob o ponto de vista do conjunto das causas externas, o valor pago por dia de internação foi de $\mathrm{R} \$ 120,00$, com algu- mas variações importantes. Os dois tipos mais freqüentes de causas que levaram à internação (quedas e acidentes de transporte) apresentaram valores acima da média. Os homicídios encontram-se também nessa situação, sendo que representaram a causa externa de custo-dia mais elevado.

Examinando os gastos médios por tipo de causa externa, naqueles que tiveram alta hospitalar a variação foi de $\mathrm{R} \$ 165,53$ (envenenamentos) a R $\$ 721,15$ (acidentes de transporte). Em todos os tipos, o gasto médio daqueles que foram a óbito foi, pelo menos, o dobro dos que tiveram alta hospitalar (Tabela 3).

Sob a ótica da natureza da lesão (Tabela 4), constatou-se que os traumas que atingiram segmentos definidos ou múltiplos segmentos em geral tiveram valores superiores à média, que foi de $\mathrm{R} \$ 119,97$ (praticamente o mesmo valor apresentado na Tabela 3). $\mathrm{O}$ inverso ocorreu em relação aos demais tipos de lesões, excetuando-se a geladura (R\$ 134,71) e as complicações de cuidados médicos (R\$132,35).

Tais resultados apontam, mais uma vez, para a estreita relação existente entre os tipos de causas externas mais freqüentes e o trauma resultante. Neste último, o bloco "traumatismo" aparece como um grande conjunto predominante, diferenciado pelo tipo de saída hospitalar - alta ou óbito - que pode trazer embutido o nível de gravidade do trauma.

Este fenômeno se repete ao se analisar o gasto médio com as internações do SUS, seja do ponto de vista dos tipos de causas externas, seja pela natureza das lesões (Tabelas 3 e 4). O gasto médio em reais quase triplicou, quando da vigência de óbito, nas duas abordagens. Na geladura não houve ocorrência de óbito e, na realidade, a avaliação ficou prejudicada na medida em que estiveram, nessa condição, apenas dois pacientes.

\section{Considerações finais, conclusões e algumas recomendações}

Embora algumas limitações estejam presentes no Banco de Dados relativo às 
Tabela 3 - Gasto médio e custo-dia (em Reais) das internações por causas externas segundo tipo de causa e tipo de saída, Estado de São Paulo, 2000.

Table 3 - Average expenditures and cost per day (in reals) of hospital admissions due to external causes according to cause and discharge status, State of São Paulo, 2000.

\begin{tabular}{lccc}
\hline Tipo & \multicolumn{2}{c}{ Gasto Médio (em Reais) } & $\begin{array}{c}\text { Custo dia } \\
\text { (em Reais) }\end{array}$ \\
\hline Quedas & 485,40 & $1.271,18$ & 122,55 \\
Acidentes de transporte & 721,15 & $1.500,79$ & 132,18 \\
Envenenamento & 165,53 & 562,83 & 62,39 \\
Queimaduras & 649,43 & $1.620,27$ & 90,80 \\
Penetração de corpo estranho & 204,00 & 644,57 & 96,40 \\
Acidente por arma de fogo & 572,93 & $1.137,39$ & 119,58 \\
Demais acidentes & 534,70 & $1.773,87$ & 116,57 \\
Suicídios e tentativas & 313,69 & 801,95 & 78,64 \\
Homicídios e tentativas & 672,48 & $1.338,08$ & 133,14 \\
lgnorado & 446,40 & $1.332,40$ & 104,48 \\
Seqüelas & 542,75 & $1.182,34$ & 78,79 \\
Fatores suplementares & 462,47 & 955,31 & 91,85 \\
\hline Total & 537,69 & $1.358,45$ & 120,00 \\
\hline
\end{tabular}

Tabela 4 - Gasto médio e custo-dia (em Reais) das internações SUS por causas externas segundo natureza da lesão e tipo de saída, Estado de São Paulo, 2000.

Table 4 - Average expenditures and cost per day (in Reais) of SUS hospital admissions due to external causes according to type of injury and discharge status, State of São Paulo, 2000.

\begin{tabular}{|c|c|c|c|}
\hline \multirow[t]{2}{*}{ Natureza da lesão } & \multicolumn{2}{|c|}{ Gasto Médio (em Reais) } & \multirow{2}{*}{$\begin{array}{l}\text { Custo dia } \\
\text { (em Reais) }\end{array}$} \\
\hline & Alta & Óbito & \\
\hline \multicolumn{4}{|l|}{ Traumatismo } \\
\hline Cabeça & 513,14 & $1.255,33$ & 122,83 \\
\hline Pescoço & 824,42 & $2.192,64$ & 105,82 \\
\hline Tórax & 640,95 & $1.239,54$ & 111,98 \\
\hline Abdômen & 778,97 & $1.497,17$ & 126,39 \\
\hline Membro Superior & 335,23 & $1.257,78$ & 125,72 \\
\hline MI-1 (quadril e coxa) & $1.053,70$ & $1.468,63$ & 146,91 \\
\hline MI-2 (joelho, perna e pé) & 529,11 & $1.694,68$ & 117,84 \\
\hline Múltiplos & 704,99 & $1.514,82$ & 131,05 \\
\hline Não especificado & 307,42 & 945,25 & 73,94 \\
\hline Corpo Estranho & 160,10 & 524,89 & 92,45 \\
\hline Queimaduras & 758,62 & $1.881,57$ & 93,91 \\
\hline Geladura & $1.616,69$ & - & 134,71 \\
\hline Intoxicações & 172,65 & 584,95 & 58,42 \\
\hline Outros efeitos & 189,81 & 652,40 & 56,73 \\
\hline Complicações precoces traum. & 392,97 & $1.122,15$ & 68,25 \\
\hline Complicações de cuidados méd. & 807,19 & $2.170,89$ & 132,35 \\
\hline Seqüelas & 583,46 & 744,82 & 67,43 \\
\hline$\overline{\text { Total }}$ & 542,97 & $1.376,66$ & 119,97 \\
\hline
\end{tabular}


internações hospitalares do Sistema Único de Saúde no Brasil (SIH-SUS), parece ser possível afirmar, como já fizeram outros autores, que os dados, cerca de 12 milhões de internações, permitem uma boa visão sobre a morbidade hospitalar no país. O trabalho, que teve por objetivo conhecer as hospitalizações decorrentes de lesões e envenenamentos em hospitais próprios ou conveniados com o SUS no Estado de São Paulo, adotou metodologia específica de trabalho, permitindo chegar a algumas conclusões importantes.

Estudando as causas naturais de um lado (excluídas as internações por problemas ligados a gravidez, parto e puerpério) e as externas de outro, foi possível mostrar, tanto para São Paulo quanto para o Brasil, que os acidentes e violências, que representam menos de $10 \%$ do total de internações, têm um tempo de permanência menor do que as causas naturais e gastam cerca de $10 \%$ do valor total pago para todas as internações.

\section{Entretanto,}

- O custo médio (em Reais) despendido para o pagamento das hospitalizações por causas externas é maior que o de causas naturais (19\% no Brasil e cerca de 5\% em São Paulo) e

- O custo-dia é também mais elevado para o valor das causas externas $(47,4 \%$ no Brasil e 50,6\% em São Paulo).

Assim, pelos resultados aqui apresentados, pode-se afirmar que os gastos do SUS medidos pelo custo-dia e pelo gasto-médio com as internações hospitalares por causas externas são superiores àqueles decorrentes de causas naturais, seja no âmbito do Brasil, seja no Estado de São Paulo, embora o tempo médio de internação em dias seja menor em ambas as áreas. Além disso, constatou-se que as internações no Estado de São Paulo foram preponderantemente decorrentes de quedas, seguidas pelos acidentes de transporte e que, em geral, apresentaram custo-dia e gasto médio superior às médias obtidas. Assim, a necessidade de melhorar as atividades de prevenção destes agravos fica fortemente indicada, a fim de interromper a cadeia de eventos antes de sua ocor- rência e/ou minimizar os efeitos deletérios subseqüentes. Os resultados obtidos mostram ainda que, na análise segundo natureza da lesão, os que faleceram durante a internação apresentaram gastos triplicados se comparados aos que tiveram alta, o que fala a favor de maior gravidade de lesão nos pacientes do primeiro grupo.

Outrossim, para melhor especificar e aprimorar tais atividades, é importante que haja uma análise pormenorizada dos tipos de causas, particularmente as quedas e os acidentes de transporte, relacionando-os com as variáveis sexo e idade.

Sugere-se também que os estudos tenham prosseguimento, estabelecendo comparações com a mortalidade pelas mesmas causas, a fim de que possam ser conhecidas taxas específicas de letalidade segundo o tipo de causas.

Por outro lado, uma tabela detalhada, relativa ao cruzamento das variáveis "natureza da lesão" e "tipo de causa externa” mostra-se absolutamente fundamental para que problemas vários possam ser melhor esclarecidos.

Cumpre também lembrar que a proporção de internados devido a causas externas deve corresponder a cerca de $20 \%$ de todas as vítimas destas causas, o que requer, urgentemente, estudo dos pacientes atendidos em Serviços de Emergências. No momento, o perfil das vítimas atendidas e a seguir liberadas nesses Serviços não pode sequer ser recuperado, na medida em que não há banco de dados universais disponibilizados de forma sistematizada e com coleta contínua. Este é também um aspecto que precisa ser resolvido a fim de possibilitar uma melhor compreensão das ocorrências de causas externas e intervenções em trauma no nosso meio.

Somente com a conjugação desses esforços e análises contínuas da situação será possível investir, com maior segurança, nos aspectos ligados à prevenção primária, com abordagens que visem a evitar as causas externas antes que elas ocorram; à prevenção secundária, com abordagens que tenham como foco as respostas mais imediatas à violência lato senso, tais como a assistência 
pré-hospitalar, serviços de emergência e hospitalar propriamente dito; e à prevenção terciária, com abordagens que visem à as- sistência a longo prazo, como reabilitação e reintegração, para tentar diminuir o trauma ou reduzir as internações ${ }^{10}$.

\section{Referências}

1. Champion HR et al. The major trauma outcome study: estabilishing national norms for trauma care. J Trauma 1990; 30(11): 1356-65.

2. Organização Mundial da Saúde - Classificação Internacional de Doenças - 10a Revisão. CBCD; 1995.

3. Koizumi MS, Lebrão ML, Mello Jorge MHP, Primerano V. Morbimortalidade por traumatismo crânioencefálico no município de São Paulo, 1997. Arq Neuropsiquiatr 2000; 58(1): 81-9.

4. Koizumi MS, Mello Jorge MHP, Nóbrega LRB, Waters C. Crianças internadas por traumatismo crânioencefálico, no Brasil, 1998: causas e prevenção. IESUS 2001; 10(2): 93-101.

5. Mello Jorge MHP et al. Acidentes e violência no Brasil. Análise dos dados de mortalidade. Rev Saúde Pública 1997; 31 (S4): 5-25.

6. Gawryszewski VP, Mello Jorge MHP. Mortalidade violenta no município de São Paulo nos últimos 40 anos. Rev Bras. Epidemiol 2000; 3 (1-3): 50-69.

7. Levcovitz E, Pereira TRC. SIH/SUS (Sistema AIH): uma análise do sistema público de remuneração das internações hospitalares no Brasil, 1983-1991. Rio de Janeiro, UERJ; 1993.

8. Risi Jr JB et al. Indicadores Básicos para a saúde no Brasil: conceitos e aplicações. RIPSA: Brasília; 2002.

9. Lebrão MC, Mello Jorge MHP, Laurenti R. Morbidade hospitalar por lesões e envenenamentos. Rev Saúde Pública 1997, 31 (S4): 26-37.

10. Organização Mundial de Saúde. Relatório mundial sobre violência e saúde OMS, Genebra, 2002. Tradução: Secretaria de Estado dos Direitos Humanos, Ministério da Justiça; Brasil; 2002.

11. Iunes RF. Impacto econômico das causas externas no Brasil: um esforço de mensuração. Rev Saúde Pública 1997; 31(S4): 38-46.

12. Instituto de Pesquisa Econômica Aplicada - Impactos sociais e econômicos dos acidentes de trânsito nas aglomerações urbanas - síntese da pesquisa. Brasília; 2003.
13. Brandt MM, Ahrns KS, Corpron CA, Flanklin GA, Wahl $\mathrm{WL}$. Hospital cost is reduced by motorcycle helmet use. J Trauma 2002; 53(3): 469-71.

14. Dueck A, Poenaru D, Pichora DR. Cost factors in Canadian pediatric trauma Can J Surg 2001; 44(2): 11721.

15. Deslandes SF, Silva CMFP, Ugá MAD. O custo do atendimento emergencial às vítimas de violências em dois hospitais do Rio de Janeiro. Cad Saúde Pública 1998; 14(2): 287-99.

16. Feijó MCC, Portela MC. Variação no custo de internações hospitalares por lesões: os casos dos traumatismos cranianos e acidentes por arma de fogo. Cad Saúde Pública 2001; 17(3): 627-37.

17. Max W, Stark B, Root S. Putting a lid on injury costs: the economic impact of the California motorcycle helmet law. J Trauma 1997; 45(3): 5-25.

18. McGarry LJ et al. Outcomes and costs of acute treatment of traumatic brain injury. J Traum 2002; 53(6): 1152-9.

19. Nurmi I, Luthje P. Incidence and costs of falls and fall injuries among elderly in institutional care. Scand $J$ Prim Health Care 2002; 20(2): 118-22.

20. Iunes, RF. The economic impact of violence on health care services: a case study of the city of São Paulo. S Paulo, 1994 (trabalho elaborado para OPAS/OMS, 1994 - Mimeo).

21. Ugá MAD. Impacto económico de la violencia sobre el servicio de salud en países de América Latina y Caribe: el caso de Rio de Janeiro, 1994. (trabalho elaborado para a OPAS/OMS, cit. em Iunes, ref. 9).

22. Ministério da Saúde, Datasus: SIH, 2000 (CD-Rom). recebido em: 09/03/04 versão reformulada apresentada em: 12/05/04 aprovado em: 17/05/04 